\title{
Application of the k-medoids Partitioning Algorithm for Clustering of Time Series Data
}

DOI:

10.1109/ISGT-Europe47291.2020.9248796

Link to publication record in Manchester Research Explorer

\section{Citation for published version (APA):}

Radovanovic, A., Ye, X., Milanovic, J. V., Milosavljevic, N., \& Storchi, R. (2020). Application of the k-medoids Partitioning Algorithm for Clustering of Time Series Data. 645-649. Paper presented at 2020 IEEE PES Innovative Smart Grid Technologies Conference Europe (ISGT-Europe), The Hague, Netherlands. https://doi.org/10.1109/ISGT-Europe47291.2020.9248796

\section{Citing this paper}

Please note that where the full-text provided on Manchester Research Explorer is the Author Accepted Manuscript or Proof version this may differ from the final Published version. If citing, it is advised that you check and use the publisher's definitive version.

\section{General rights}

Copyright and moral rights for the publications made accessible in the Research Explorer are retained by the authors and/or other copyright owners and it is a condition of accessing publications that users recognise and abide by the legal requirements associated with these rights.

\section{Takedown policy}

If you believe that this document breaches copyright please refer to the University of Manchester's Takedown Procedures [http://man.ac.uk/04Y6Bo] or contact uml.scholarlycommunications@manchester.ac.uk providing relevant details, so we can investigate your claim.

\section{OPEN ACCESS}




\section{Application of the k-medoids Partitioning Algorithm for Clustering of Time Series Data}

\author{
Ana Radovanović, Student Member \\ IEEE \\ Department of Electrical and Electronic \\ Engineering \\ The University of Manchester \\ Manchester, UK
na.radovanovic@manchester.ac.uk \\ Manchester, UK
ana.radovanovic@manchester.ac.uk
}

\author{
Nina Milosavljević \\ Division of Neuroscience and Experimental Psychology \\ The University of Manchester \\ Manchester, UK \\ nina.milosavljevic@manchester.ac.uk
}

\author{
Jovica V. Milanović, Fellow IEEE \\ Department of Electrical and Electronic \\ Engineering \\ The University of Manchester \\ Manchester, UK \\ milanovic@manchester.ac.uk
}

\begin{abstract}
Data clustering has been widely applied in numerous areas in order to pave the way for adequate and efficient modelling, control and operation. In the past, most of the data clustering was carried out on static data. However, wider application of time series data has increased the need for time series clustering techniques. This paper presents a comprehensive analysis of the applicability of a standard clustering algorithm, the k-medoids algorithm, for clustering of two diverse time series datasets. The k-medoids algorithm is tested on dynamic power responses of a hybrid renewable energy source plant and neuroscience spike-train data. The main stages in clustering process, that is, data processing, the selection of the optimal distance measure and the estimation of the optimal number of clusters, are analyzed in detail.
\end{abstract}

Keywords-data mining, $k$-medoids algorithm, electric power system, neuroscience, time series clustering

\section{INTRODUCTION}

Data clustering, a data mining method, represents the process of unsupervised partitioning of a dataset into groups, so-called clusters, according to the similarity between its elements [1], [2]. The basic objective of data clustering is to ensure that the objects in a certain cluster are more similar to each other than to the objects in other clusters, that is, intra-cluster similarity should be higher than inter-cluster similarity. Past work in the area of data clustering was mainly focused on the clustering of static data (data whose features remain unchanged or change negligibly over time) [3], [4]. However, due to the growing ability for data storage, data from various areas ranging from sales data and stock prices to biomedical and electrical power system measurements can be recorded and stored as time series data for a long period. A need to analyze these datasets efficiently has resulted in an increasing interest in time series clustering techniques.

Time series clustering can be performed on raw data or feature-/model-based representations of temporal sequences [3]. Both approaches are based on standard clustering methods originally designed for static data. Raw data-based method uses standard clustering algorithms in combination with distance measures more adequate for temporal sequences. On the other hand, feature-based time series clustering is based on transforming time series data into static data utilizing feature extraction methods, whereas model-based clustering methods

\author{
Riccardo Storchi \\ Division of Neuroscience and Experimental Psychology \\ The University of Manchester \\ Manchester, UK \\ riccardo.storchi@manchester.ac.uk
}

represent temporal sequences by the chosen mathematical model. Standard clustering methods with distance measures suitable for static data are then applied to transformed time series. Basic clustering algorithms, partitioning (among them the most popular are the k-means and k-medoids) and hierarchical, have been most widely used in time series clustering, even though various other algorithms, such as density-based, model based, grid-based, neural networkbased, etc., have emerged due to a growing need for solving different clustering tasks [2], [5].

In this research both of these methods were tested namely, k-medoids, as the most widely used partitioning algorithm, and the hierarchical agglomerative clustering algorithm to assess their suitability for clustering time series data from different fields of study. This paper presents the results of the comprehensive study of the adequacy of using the k-medoids clustering algorithm for time series data clustering. The analysis addresses a major issue in the application of the kmedoids algorithm - the selection of the adequate distance measure and the optimal number of clusters. Two different datasets from electric power system and neuroscience area are used for testing the performance of the k-medoids clustering algorithm. Testing the clustering method on diverse temporal sequences provides more reliable assessment of its suitability for time series clustering tasks. Furthermore, as test datasets correspond to time series data frequently analyzed in power system and neuroscience research domains [6], [7], the results obtained in the study can be used as a starting point when carrying out data clustering in these or other research areas when dealing with similar structures of datasets.

\section{Clustering Procedure}

\section{A. k-medoids Clustering Algorithm}

The basic idea of the k-medoids partitioning algorithm is to perform division of a dataset into a pre-defined $k$ number of clusters with the aim of minimizing the average distance between a clustering object and its cluster's representative [2]. The representatives of clusters, i.e., medoids, are selected among the clustering objects. The clustering procedure starts with an arbitrary selection of initial medoids among all clustering objects. Following this, the rest of the data are allocated to the closest medoid according to the selected distance measure. In the following steps, the algorithm checks 
whether the substitution of an already chosen medoid by a non-representative object will reduce the clustering objective function [2]. The iterative procedure continues until the value of the clustering objective function becomes stable. One of the drawbacks of the k-medoids algorithm is that the result may not necessarily represent the global minimum [2]. Thus, the clustering procedure is usually repeated several times with different initial medoids and the clustering result that corresponds to the smallest value of the objective function is chosen as the final output of the algorithm [2]. The k-medoids clustering algorithm is more robust to noise data and outliers than the k-means partitioning method. However, these features come with higher computational complexity $O\left(k(N-k)^{2}\right)$, where $N$ is the number of clustering objects, compared to $O(N)$ for the k-means algorithm [2].

The selection of distance measure has a significant influence on the algorithm's outputs as it defines how the similarity between clustering objects is calculated. Distance measures commonly used in time series clustering are the Euclidean, Pearson's correlation, Spearman and Dynamic Time Warping (DTW) distance [1], [3], [8]. The mathematical expressions for the first three distance measures are as follows:

$$
\begin{gathered}
d_{\text {Euclidean }}=\sqrt{\sum_{i=1}^{n}\left(x_{i}-y_{i}\right)^{2}}, \\
d_{\text {Pearson }}=1-\frac{\sum_{i=1}^{n}\left(x_{i}-\bar{x}\right)\left(y_{i}-\bar{y}\right)}{\sqrt{\sum_{i=1}^{n}\left(x_{i}-\bar{x}\right)^{2}} \sqrt{\sum_{i=1}^{n}\left(y_{i}-\bar{y}\right)^{2}}}, \\
d_{\text {Spearman }}=1-\frac{\sum_{i=1}^{n}\left(r_{x, i}-\overline{r_{x}}\right)\left(r_{y, i}-\overline{r_{y}}\right)}{\sqrt{\sum_{i=1}^{n}\left(r_{x, i}-\overline{r_{x}}\right)^{2}} \sqrt{\sum_{i=1}^{n}\left(r_{y, i}-\overline{r_{y}}\right)^{2}}},
\end{gathered}
$$

where $x$ and $y$ are time sequences, $n$ is the number of data samples, $\bar{x}$ and $\bar{y}$ are the mean values of $x$ and $y, r_{x}$ and $r_{y}$ are rank vectors of $x$ and $y$, and $\overline{r_{x}}$ and $\overline{r_{y}}$ are the mean values of $r_{x}$ and $r_{y}$.

These three distance measures belong to a group of lock-step distances as they are based on the distances between data samples occurring at the same time steps. On the other hand, the DTW distance allows matching of samples that are shifted in time [3]. Namely, the DTW distance is based on an $n \times m$ matrix, where $n$ and $m$ are dimensions of the analysed time series [9]. The matrix contains the Euclidean distances between each pair of data samples from the time series. The aim is to find a sequence of matrix elements (so-called warping path) that corresponds to the minimum distance between the time series. The optimal warping path is determined using dynamic programming. The warping path has to begin with a pair of the first elements of the time series and ends with a pair of the last elements. Also, the elements of the warping path have to be adjacent matrix elements and the indices of paired elements have to be in non-monotonically decreasing order. However, the computational complexity is much higher than in the case of the considered lock-step distances - $O\left(n^{2}\right)$ compared to $O(n)$ [10].

\section{B. Selection of the Optimal Number of Clusters}

As aforementioned, the number of clusters has to be defined prior to carrying out the clustering process. Optimal number of clusters is typically determined using internal clustering evaluation indices, which are based on the assessment of intra-cluster and inter-cluster similarity [2], [4]. The k-medoids algorithm is repeated for different number of clusters and the selected clustering evaluation index is calculated for each clustering result. The final number of clusters is determined based on the optimal value of the index. Some of the most common internal clustering evaluation indices are:

- Mean square error (MSE): The MSE index calculates the average distance between an object and its corresponding cluster representative [11]:

$$
M S E=\frac{1}{N} \sum_{i=1}^{N} d^{2}\left(x_{i}, w_{j: x_{i} \in \Omega_{j}}\right),
$$

where $x_{i}$ is the $i$-th clustering object, $w_{j}$ is a representative of the $j$-th cluster, $\Omega_{j}$ is a set of elements in the $j$-th cluster, $d()$ is a distance measure.

- Mean index adequacy (MIA): The MIA index, similar to the MSE index, is calculated based on the average distances between clustering objects and their cluster representatives [12]:

$$
\begin{gathered}
M I A=\left(\frac{1}{k} \sum_{j=1}^{k} d^{2}\left(w_{j}, \Omega_{j}\right)\right)^{1 / 2}, \\
d^{2}\left(w_{j}, \Omega_{j}\right)=\frac{1}{N_{j}} \sum_{x_{i} \in \Omega_{j}} d^{2}\left(w_{j}, x_{i}\right),
\end{gathered}
$$

where $N_{j}$ is the number of elements in the $j$-th cluster.

- Within cluster sum of squares to between cluster variation ratio (WCBCR): The WCBCR is calculated based on the distances between each clustering object and its corresponding cluster representative and the distances between cluster representatives [13]:

$$
W C B C R=\frac{\sum_{j=1}^{k} \sum_{x_{i} \in \Omega_{j}} d^{2}\left(x_{i}, w_{j}\right)}{\sum_{1 \leq q<p}^{k} d^{2}\left(w_{p}, w_{q}\right)} .
$$

Smaller values of all three internal indices indicate more compact and separated clusters. The elbow method is usually used for estimating the optimal number of clusters [13]. The method is based on plotting the values of the selected index against the number of clusters and the best number of clusters is located at the knee of the curve. The knee of the curve is commonly estimated using the method described in [13]. The two-tangent method is based on visual inspection of the knee location and drawing tangents to the parts of the curve on either side of the estimated knee location. The knee of the curve is then located at the intersection of the tangents.

\section{RESULTS AND DISCUSSION}

The k-medoids clustering algorithm is tested on two datasets from electric power systems and neuroscience areas. Four distance measures (the Euclidean, Pearson's correlation, Spearman and DTW) and three clustering evaluation indices (MSE, MIA and WCBCR) are utilized for assessing the performance of the algorithm. Data processing and clustering procedures are carried out in Matlab R2019a software environment [14].

\section{A. Case Study I: Power System Dataset}

The power system dataset consists of active power responses of a hybrid renewable energy source plant to an 


\section{ACCEPTED VERSION OF THE PAPER}

external three-phase self-clearing fault. The hybrid power plant contains a wind farm, a photovoltaic, hydro, biomass and biogas plant and a battery energy storage system. All individual plants have the same nominal capacity (210 MVA) and are connected to a common $230 \mathrm{kV}$ transmission network bus. Simplified representation of transmission system in the form of two parallel lines and a single-machine infinite bus is used in the study. The dynamic behavior of the plant was investigated for 1,600 hybrid plant operating conditions. The responses were recorded for a period of $9 \mathrm{~s}$ after the fault clearing (fault duration was $100 \mathrm{~ms}$ ) and with a sampling rate of $10 \mathrm{~ms}$. As the focus of clustering task is on similarity in shape of power responses, the responses have to be represented on a common scale. Given the total plant power output was not the same in all analyzed operating points, z-normalization of the responses was carried out before conducting the clustering procedure [15]:

$$
z(t)=\frac{P(t)-P_{\text {mean }}}{S D(P(t))},
$$

where $z(t)$ is a z-normalized power response in p.u, $P(t)$ is a power response in $\mathrm{MW}, P_{\text {mean }}$ is the average of the whole response, $S D(P(t))$ is a standard deviation of the response.

Fig. 1 - Fig. 3 present the change of three internal clustering evaluation indices (MSE, MIA and WCBCR) with the number of clusters for four analyzed distance measures. As expected, different distance measures produce different curves for the same clustering index. Also, different clustering indices suggest different number of clusters for the same distance measure. The WCBCR index results in the most regular L-shaped curves, whereas the curves for the MIA index have the most irregular shape. Thus, the WCBCR indicator is used for estimating the number of clusters in the active power dataset. According to this index, the best number of clusters for all the considered distances is four, except for the Spearman distance which requires three clusters.

The Euclidean and Pearson's correlation distances result in identical clustering results, which are shown in Fig. 4 and Fig. 5. Cluster 3 has a very low level of compactness as it consists of two groups of responses having significantly different amplitude and damping. The group of responses with larger amplitude is similar to responses in cluster 2, i.e., it should have been allocated to this cluster. Also, the quality of cluster 1 is not satisfactory as it contains a group of responses being out-of-phase with respect to the majority of the responses (see the zoomed part of cluster 1 in Fig. 4). On the other hand, clusters 2 and 4 are compact, but similar to each other.

When it comes to the three clusters produced by the Spearman distance measure, they can be obtained on the basis of the clusters presented in Fig. 4 and Fig. 5. Namely, one of the clusters is a combination of clusters 2 and 4 , while the remaining two clusters correspond to clusters 1 and 3, respectively.

Unlike in the case of the lock-step distances, the DTW distance identifies a group of highly damped responses (the group of responses with smaller amplitude in cluster 3 in Fig. 5 ) as an individual cluster. The second cluster corresponds to a major group of the responses in cluster 1 in Fig. 4, while the third is identical to cluster 4 in Fig. 5. As the remaining power responses are grouped into a single cluster, this cluster is characterized by responses being out-of-phase (similar to cluster 1 in Fig. 4).

Therefore, as none of the considered distance measures provides absolutely "well separated and compact clusters" [10] and the DTW distance is the only one that does not produce cluster 3 in Fig. 5, this distance measure can be considered as the most adequate for power system dataset clustering.

\section{B. Case Study II: Neuroscience Dataset}

The dataset used in case study II consists of neuroscience spike-train data that represent reactions of 1,492 neurons to light stimuli [16]. Spike-train data were recorded as frequencies of neuron spikes in 10-milisecond intervals (bins). Each neuron was exposed to light stimuli 80 times and each exposure (trial) lasted for 10.99 seconds. A clustering object is thus a sequence of neuron reactions to all 80 trials.

As the dataset size is not adequate for the k-medoids algorithm from the point of view of computational time, the data had to be processed before conducting the clustering process. For each neuron, a sequence of 80 trials was divided into two parts corresponding to the first and last 40 trials as the features of these two groups of trials are considerably different and neuron responses belonging to the same group of trails were summed. In this way the dataset size was reduced from $1,492 \times(80 \times 1,099)$ to $1,492 \times(2 \times 1,099)$. Furthermore, the original neuroscience dataset is characterized by a large number of zeros corresponding to neuron inactivity. In order to avoid singularity problems when calculating similarity between the clustering objects using (2) and (3), the data were Gaussian filtered (the parameters of Gaussian filter are $L=30, \sigma=3$ ). Final step in data processing relates to z-normalization of neuron spikes as the aim is to cluster the data based on the similarity in shape (as in case study I).
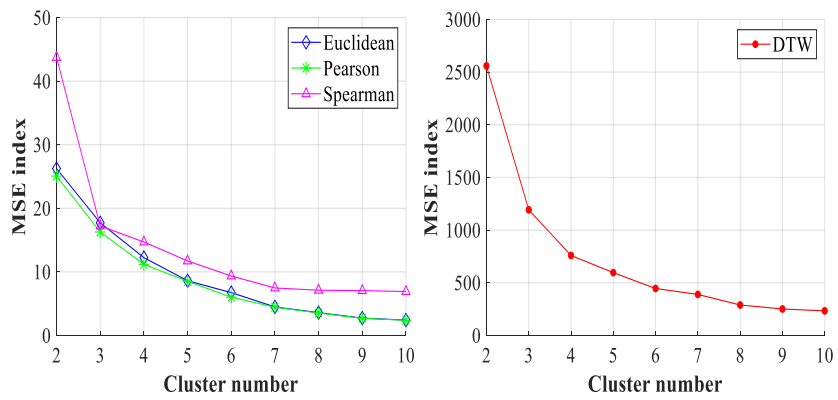

Fig. 1. Power system dataset: MSE index for the analyzed distance measures.
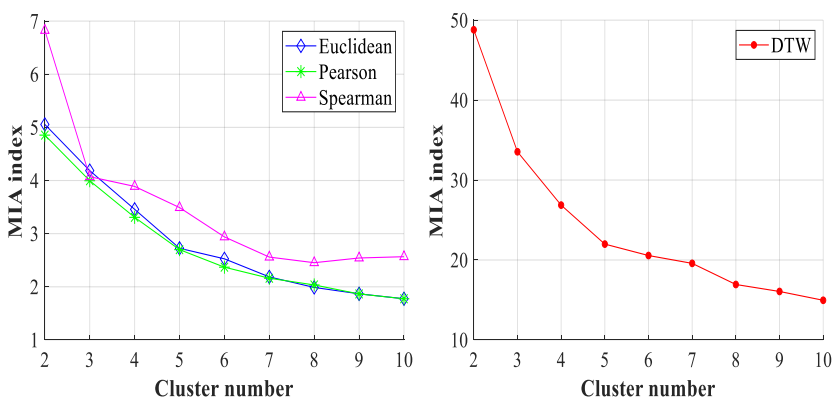

Fig. 2. Power system dataset: MIA index for the analyzed distance measures. 
Curves corresponding to each combination of the analyzed clustering indices and distance measures are presented in Fig. 6 - Fig. 8. All three lock-step distance measures produce almost identical changes of the clustering indices with the number of clusters. The MSE and MIA indices are characterized by curves without noticeable knee which makes the estimation of the best number of clusters difficult and less accurate. Therefore, the WCBCR index is chosen for determining the optimal number of clusters, as in previous case study. According to this index, four clusters are required for each of the considered distance measure.
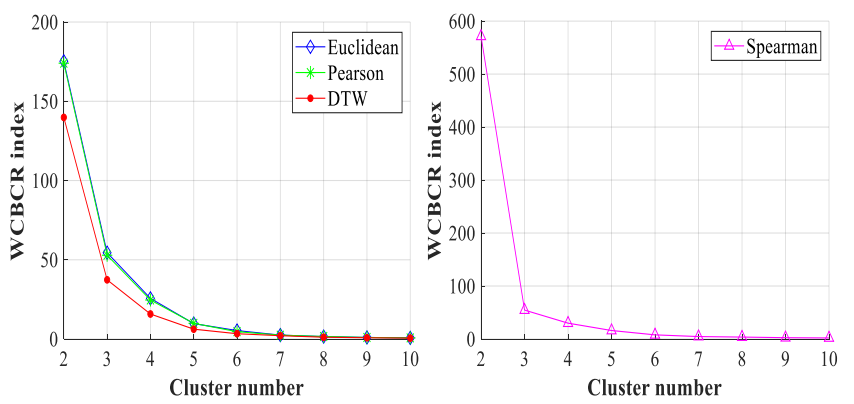

Fig. 3. Power system dataset: WCBCR index for the analyzed distance measures.
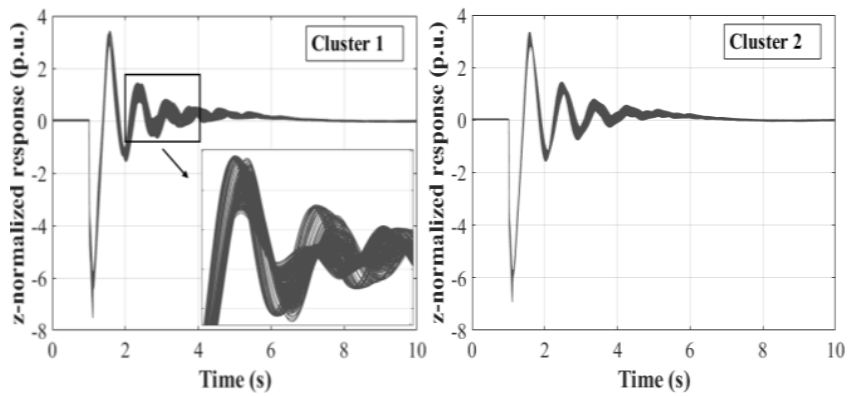

Fig. 4. Power system dataset: Cluster 1 (left) and Cluster 2 (right) in the case of the Euclidean and Pearson's correlation distances.
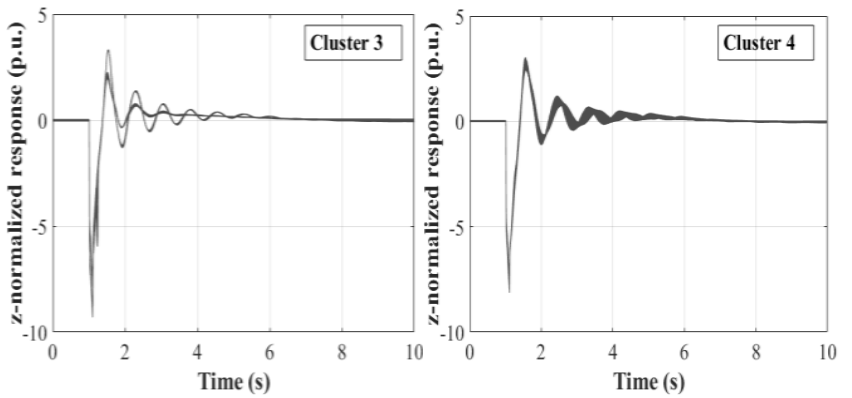

Fig. 5. Power system dataset: Cluster 3 (left) and Cluster 4 (right) in the case of the Euclidean and Pearson's correlation distances.

All four distance measures result in clusters containing groups of neurons with different firing patterns, meaning none of the analyzed distance measures in combination with the k-medoids algorithm provides highly compact clusters. As in case study I, the Euclidean and Pearson's correlation distance measures produce identical clusters. Furthermore, all three lock-step distance measures produce clusters of similar quality. Among all distances, the DTW distance is characterized by the smallest cluster having very low quality level. The worst cluster (in terms of compactness and separation from other clusters) produced by the DTW distance contains only around 70 neuron responses, whereas the worst clusters in the case of lock-step distances have more than 300 responses. Thus, the DTW distance has shown to provide the clusters of the highest quality, as in the power system case study.

Fig. 9 - Fig. 11 illustrate the worst clusters obtained in the case of each of the considered distances, while the best cluster produced by the DTW distance is shown in Fig. 12. Non-normalized spike-train data are used for presenting clustering results, as z-normalized neuron responses are not suitable for visualization of clustering results.
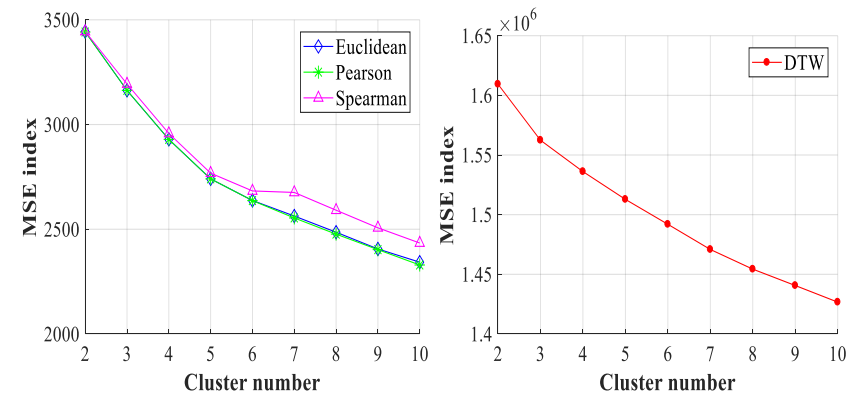

Fig. 6. Neuroscience dataset: MSE index for the analyzed distance measures.
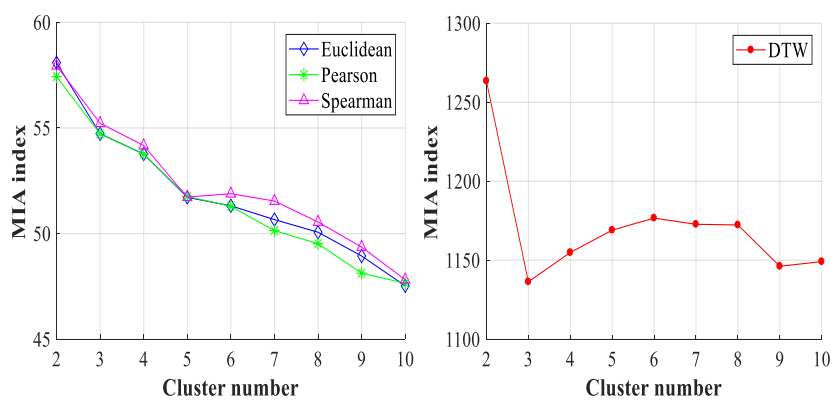

Fig. 7. Neuroscience dataset: MIA index for the analyzed distance measures.
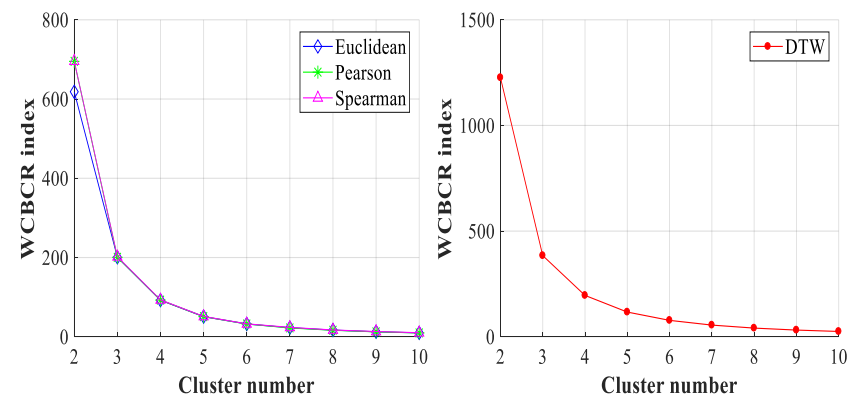

Fig. 8. Neuroscience dataset: WCBCR index for the analyzed distance measures.
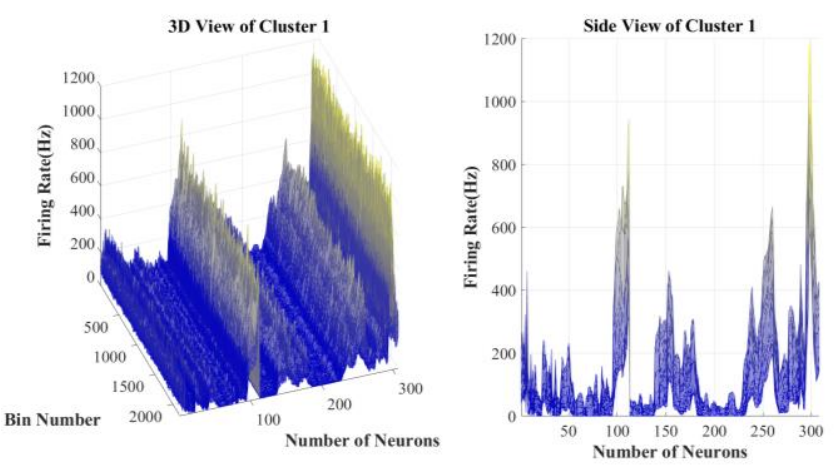

Fig. 9. Neuroscience dataset: 3D view (left) and side view (right) of the worst cluster in the case of the Euclidean and Pearson's correlation distances. 

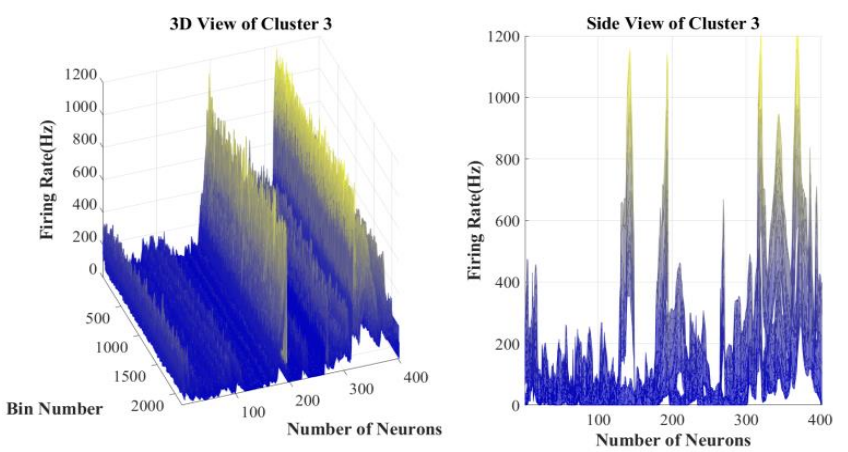

Fig. 10. Neuroscience dataset: 3D view (left) and side view (right) of the worst cluster in the case of the Spearman distance.
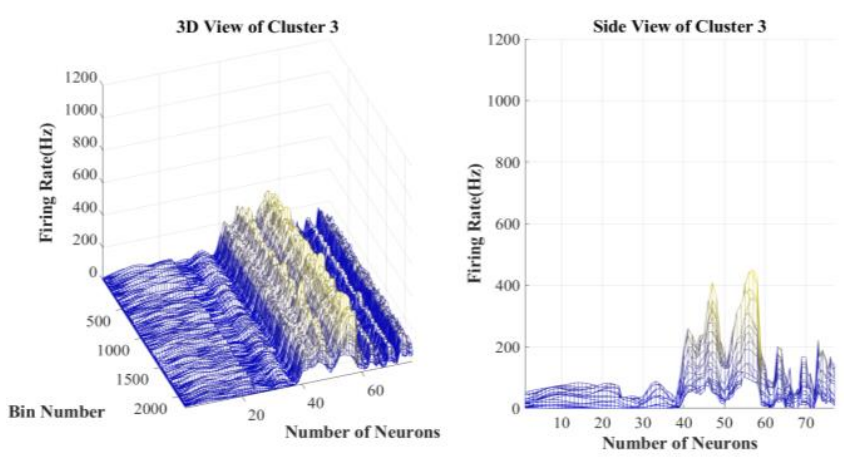

Fig. 11. Neuroscience dataset: 3D view (left) and side view (right) of the worst cluster in the case of the DTW distance.
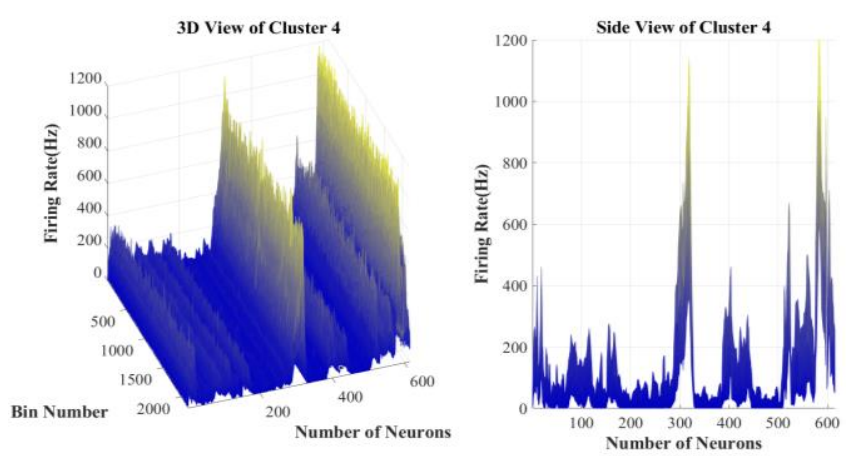

Fig. 12. Neuroscience dataset: 3D view (left) and side view (right) of the best cluster in the case of the DTW distance.

\section{CONCLUSION}

The paper explores the adequacy of the k-medoids clustering algorithm for time series clustering using power system and neuroscience datasets. Results have demonstrated that the performance of the k-medoids algorithm is affected by the choice of the distance measure to a considerable extent.

The DTW distance has outperformed the lock-step distance measures in both case studies which makes it the most suitable distance measure for both power system and neuroscience data. Furthermore, there is no difference in the performance of the Euclidean and Pearson's correlation distance measures as they produced the same clusters in both case studies.

As for clustering evaluation indices, the WCBCR index is characterized by curves with the most noticeable knee for both analyzed datasets, and thus the most adequate for estimating the optimal number of clusters. On the contrary, the curves corresponding to the MIA index have the most irregular shape making them the least suitable for estimating the optimal number of clusters using the elbow method.

Even though the k-medoids method (as an example of the partitioning methods) and the four distance measures were selected based on comprehensive literature review as the best candidates for the task in hand, the perfectly compact and separated clusters have not been obtained. Therefore, future research will focus on analyzing the suitability of other clustering algorithms and evaluation indices for clustering the time series power system and neuroscience data.

\section{ACKNOWLEDGMENT AND DISCLAIMER}

The research is supported by the EU H2020 project CROSSBOW (grant agreement 773430), Fight for Sight Fellowship 5047/5048 and National Centre for Replacement Refinement and Reduction of Animals in Research (NC3Rs) via a David Sainsbury Fellowship (NC/P001505/1). The paper reflects only the authors' views and neither the Agency nor the Commission are responsible for any use that may be made of the information contained therein.

\section{REFERENCES}

[1] A. K. Jain, M. N Murty, P. J. Flynn, "Data clustering: a review," ACM Comput. Surv., vol. 31, no. 3, pp. 264-323, September 1999.

[2] J. Han, M. Kamber and J. Pei, Data Mining Concepts and Techniques, USA: Elsevier: Waltam, 2012.

[3] T. Warren Liao, "Clustering of time series data - a survey," Pattern Recognit., vol. 38, no. 11, pp. 1857-1874, November 2005.

[4] S. Aghabozorgi, A. Seyed Shirkhorshidi, and T. Ying Wah, "Time series clustering - A decade review," Inform. Syst., vol. 53, pp. 16-38, October-November 2015.

[5] A. Nagpal, "Review based on Data Clustering Algorithms," in 2013 IEEE Conference on Information \& Communication Technologies, Thuckalay, Tamil Nadu, India, April 2013.

[6] A. Aljadeff, B. J. Lansdell, A. L. Fairhall, and D. Kleinfeld, “Analysis of neuronal spike trains, deconstructed”, Neuron, vol. 91, pp. 221-259, July 2016.

[7] CIGRE Report Working Group C4. 605, "Modelling and aggregation of loads in flexible power networks", February 2014.

[8] M. Kotlyar, S. Fuhrman, A. Ableson, and R. Somogyi, "Spearman correlation identifes statistically significant gene expression clusters in spinal cord development and injury," Neurochem. Res., vol. 27, no. 10, pp. 1133-1140, October 1992.

[9] E. Keogh and C. A. Ratanamahatana, "Exact indexing of dynamic time warping”, Knowl. Inf. Syst., vol. 7, no. 3, pp. 358-386, March 2005.

[10] Y. S. Jeong, M. K. Jeong, O. A. Omitaomu, "Weighted dynamic time warping for time series classification," Pattern Recognit., vol. 44, no. 9, pp. 2231-2240, September 2011.

[11] S. L. Everitt, M. Leese, D. Stahl, Cluster analysis, 5ht ed. London, UK: John Wiley \& Sons, Ltd., 2011.

[12] G. Chicco, R. Napoli, P. Postolache, M. Scutariu and C. Toader, "Customer characterization for improving the tariff offer," IEEE Trans. Power Syst., vol. 18, no. 1, pp. 381-387, February 2003.

[13] G. J. Tsekouras, P. B. Kotoulas, C. D. Tsirekis, E. N. Dialynas, and N. D. Hatziargyriou, "A pattern recognition methodology for evaluation of load profiles and typical days of large electricity customers," Electr. Pow. Syst. Res., vol. 78, no. 9, pp. 1494-1510, September 2008.

[14] "MATLAB and Statistics Toolbox Release 2019," The Mathworks, Inc., Natick, Massachusetts, United States.

[15] I. B. Mohamad, D. Usman, "Standardization and its effects on k-means clustering algorithm,” Res. J. Appl. Sci. Eng. Tech., vol. 6, no. 17, pp. 3299-3303, September 2013.

[16] N. Milosavljević, R. Storchi, C. G. Eleftheriou, A. Colins and R. S. Petersen, "Photoreceptive retinal ganglion cells control the information rate of the optic nerve," Proc. Natl. Acad. Sci USA, vol. 115, no. 50, pp. E11817-E11826, December 2018. 